\title{
Danger Conditions Detection by Using Acceleration Sensor in Smartphone
}

\author{
Muhammad Khairul Nizar Khairuddin ${ }^{\mathrm{a}}$, Hiromasa Tomimoto ${ }^{\mathrm{a}}$, Kazuhiro Nakamoto ${ }^{\mathrm{a}}$, \\ Shenglin $\mathrm{Mu}^{\mathrm{b}}$, Shota Nakashima ${ }^{\mathrm{a}}$, Hiroshi Nakamura ${ }^{\mathrm{a}}$, Kanya Tanaka, ${ }^{\mathrm{a}}{ }^{*}$ \\ ${ }^{a}$ Yamaguchi University \\ 2-16-1, Tokiwadai, Ube, Yamaguchi, 755-8611, JAPAN \\ ${ }^{\mathrm{b}}$ National Institute of Technology, Hiroshima College \\ 4272-1, Higashino, Osakikamijima-cho, Toyota-gun, Hiroshima, 725-0231, JAPAN \\ *Corresponding Author: ktanaka@yamaguchi-u.ac.jp
}

\begin{abstract}
As aging society has grown in Japan, people with disabilities have also increased. However, there is a very little number of people taking care of them. Thus, to reduce the burden of the caretaker, we proposed a meal assistant robot. With only a robot, we cannot know whether the user is in a safe state or not. Because of that, when using this meal assistance robot, a system that can detect the user danger conditions such as aspiration and fall-down and report it to the caretaker was proposed. The detection of the danger conditions is carried out using an Android smartphone. Aspiration was detected from the shock due to the user's cough, whereas the user's fall-down state was detected by the change of the acceleration of gravity due to the turn of the device. With the proposed system, if any problem occurs to the user, the caretaker can handle it immediately. In this paper, experiments to measure the recognition rate of the danger state were performed to confirm the effectiveness of the proposed system.
\end{abstract}

Keywords: aging society, aspiration, fall-down, acceleration sensor, Android smartphone.

\section{Introduction}

In recent years, aging society is growing in Japan. In 2015, the elderly person population has risen up to $33,920,000$. Among the total population ratio (the elderly population rate) is $26.7 \%$, and it is estimated that the elderly population rate reaches $39.9 \%$ in $2060^{(1,2)}$.
Nowadays, $56.0 \%$ of the aged household is living alone or living together with their spouse ${ }^{(2)}$. Even there are many people needs to be cared of, the employment of the caretaker is only $1,400,000$ which is $4.127 \%$ from the total of people need to be taken care $^{(3)}$. Hence, we can assume that the number of the caretaker is extremely not enough. Thus, we proposed a meal assistance robot to reduce the difficulty of the caretaker ${ }^{(4,5)}$. While the nursed person is eating, the caretaker can do other housework such as washing or cleaning. However, with only a robot, we cannot know whether the user is in a safe state or not. Therefore, a system when using the meal assistance robot, where an aspiration or fall-down accident can be detected and can report immediately to the caretaker if a problem occurs is proposed. The detection of the danger state is performed using an Android smartphone. There is a fall-down detection system built in the Android smartphone.

Fall-down detection and cough detection are widely studied and often used for the elderly people around the world, but most of the existing method approaches by using a sensor that detects only at a particular place. A specific floor pressure ${ }^{(6)}$, a camera-based sensor ${ }^{(7)}$ and infrared array sensor $^{(8)}$ are examples that often used for fall-down detection at a particular place. While system such as Mobicough $^{(9)}$ that detects a cough by using a microphone is used for the cough detection ${ }^{(9,10)}$. These types of sensor make it hardly possible to detect a fall-down accident at other places and take spaces to install. Besides that, sensor such as camera and microphone is a disturbance to the user's privacy. 
Regarding these problems, fall-down and aspiration detection system by using an Android smartphone is proposed. The proposed system used an acceleration sensor built in the Android smartphone to detect the shock and motion of the user's chest. Thus, aspiration and fall-down accidents detection is possible. Compare to other devices, smartphones can send information quickly to the caretaker if accidents such as fall-down and aspiration occur to the user.

This paper is organized as follows; Section 2 gives the details of the system. While in section 3 gives the explanation about the danger state that can able to be detected. Section 4 describes the experiment to evaluate the effectiveness of the proposed system. Lastly, section 5 gives the discussion and summary of the experiment result.

\section{Proposed System}

\subsection{System Summary}

By using an Android smartphone, the proposed system detects aspiration or fall-down accidents when having a meal supported by the meal assistance robot. Then, the information of the detected accidents will be alerted to the caretaker. The danger state detection is performed by using an acceleration sensor which is built in the Android smartphone.

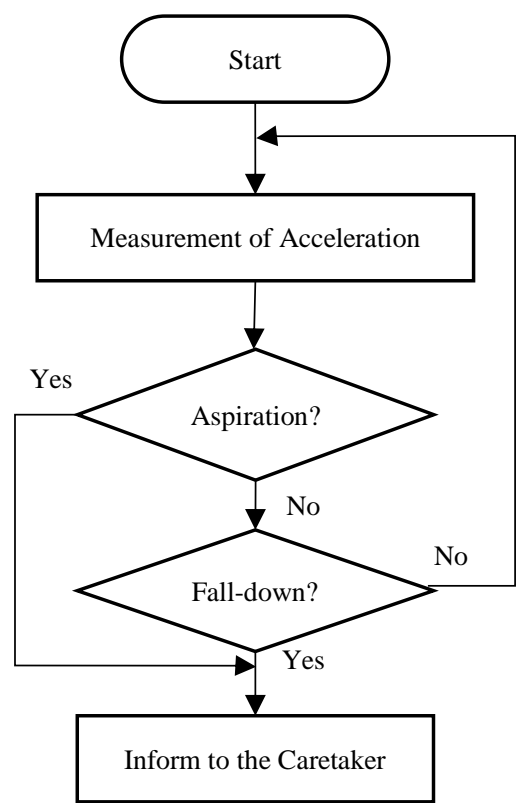

Fig. 1. The flowchart of the proposed system

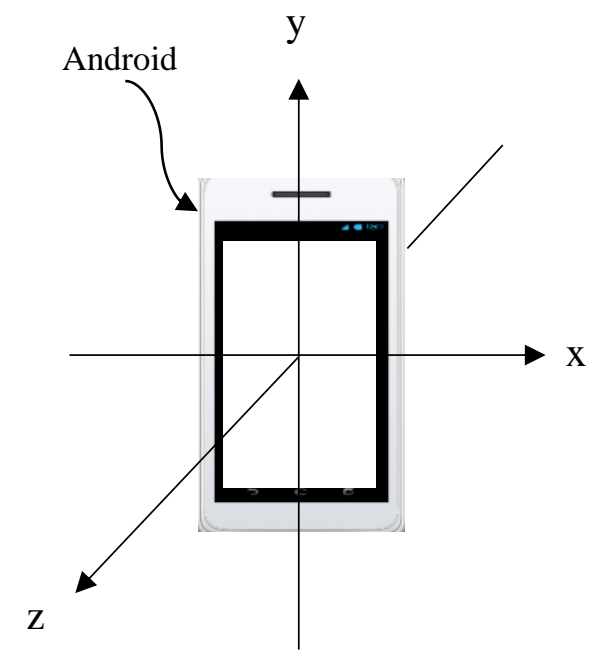

Fig. 2. Direction axes of the acceleration sensor.

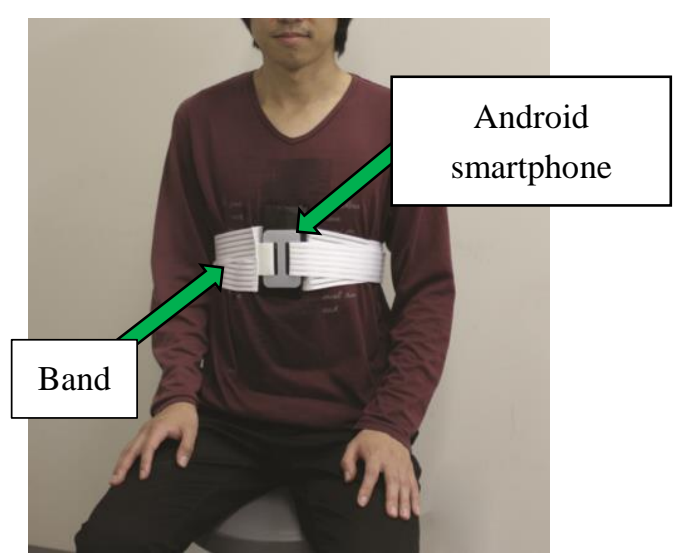

Fig. 3. Android smartphone being strapped on the user chest.

The procedure of the proposed system is shown in Fig. 1. An aspiration is detected by the shock due to the continuous of the user's cough, and the fall-down accident is detected by the change of the acceleration of gravity due to the turn of the device. The details of the danger detection will be explained respectively in section 3.2 and section 3.3 .

\subsection{Acceleration Sensor}

Acceleration sensor is generally built in a smartphone. Figure 2 shows the properties of the sensor that can measure the acceleration of 3 axes which is $\mathrm{x}$ (horizontal direction), $\mathrm{y}$ (vertical direction) and $\mathrm{z}$ (depth direction). The measurement of the acceleration sensor is included with the acceleration of gravity $g=9.8 \mathrm{~m} / \mathrm{s}^{2}$. By measuring the acceleration and carrying out the appropriate signal processing, various information such as the inclination, movement, vibration, and shock can be obtained. 


\subsection{Shock Detection by The Acceleration Sensor}

Let the measured acceleration of $\mathrm{x}$-axis (horizontal direction) be $a_{x}$, the measured acceleration of $y$-axis(vertical direction) be $a_{y}$, and the measured acceleration of z-axis( depth direction) be $a_{z}$. thus the absolute value of the compound acceleration of the 3 axes $|a|$ can be shown on the following expression.

$$
|a|=\sqrt{a_{x}{ }^{2}+a_{y}{ }^{2}+a_{z}{ }^{2}}
$$

Since the acceleration of gravity $g=9.8 \mathrm{~m} / \mathrm{s}^{2}$ is included in the measurement of acceleration, as long as the sensor is completely vertically still, the total acceleration $|a|$ is always $|a|=9.8 \mathrm{~m} / \mathrm{s}^{2}$. Moreover, when there is an exterior acceleration affecting the sensor, the change of $|a|$ occurs. Thus, the existence of a shock or the strength of the shock can be determined.

Furthermore, let the $n$-th number of data of the compound acceleration be $\left|a_{n}\right|$, thus the absolute value of the difference with (n-1)-th number of data; $\left|D_{n}\right|$ can be shown on the following expression.

$$
\left|D_{n}\right|=|| a_{n}|-| a_{n-1}||
$$

From the absolute value of the difference $|D|$, the intensity of the shock can be determined.

\section{Method of Detection}

\subsection{Measurement Condition}

The subject is as shown in Fig. 3, a band is strapped under the center of his chest, and the Android smartphone is fixed. After the subject sat down on the chair, the acceleration measurement is started. The sampling period for the acceleration sensor is $1 / 150 \mathrm{~s}$.

\subsection{Aspiration Detection}

The compound acceleration $|a|$ detected when the user is at an aspiration state while being assisted while using the meal assistance robot and after being assisted is shown in Fig. 4. However, since it is difficult to cause an aspiration intentionally, an aspiration is assumed to be determined by a period of spontaneous coughs.

As shown in Fig. 4, when being assisted, there is a change of the acceleration as the body is inclined. Furthermore, when an aspiration occurs, an intense shock due to the cough happen can be understood. To differentiate between the state of being assisted and the state when an aspiration occurs, the absolute value of the difference $|D|$ is calculated. The calculated $|D|$ is shown in Fig. 5.

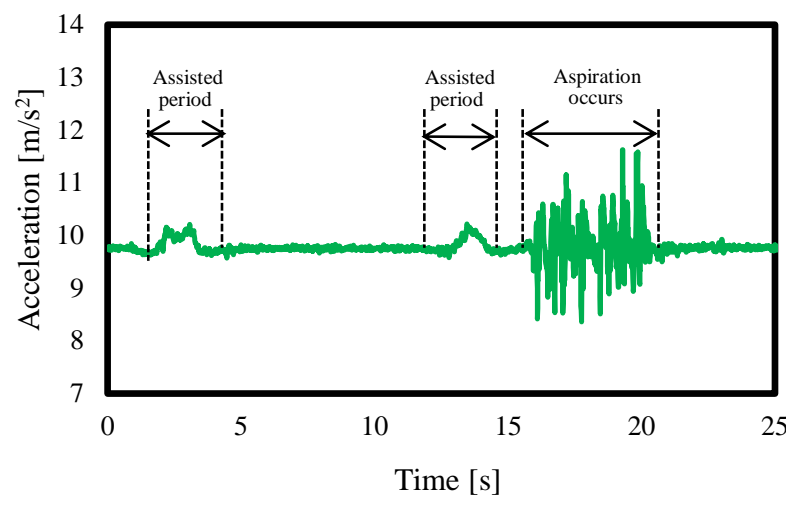

Fig. 4. Compound acceleration $|a|$ while being assisted and when aspiration occurs.

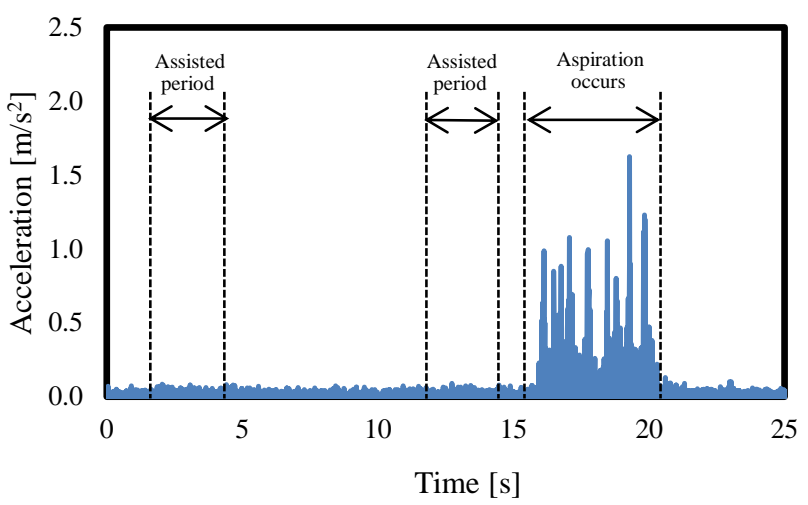

Fig. 5. Absolute value of difference $|D|$.
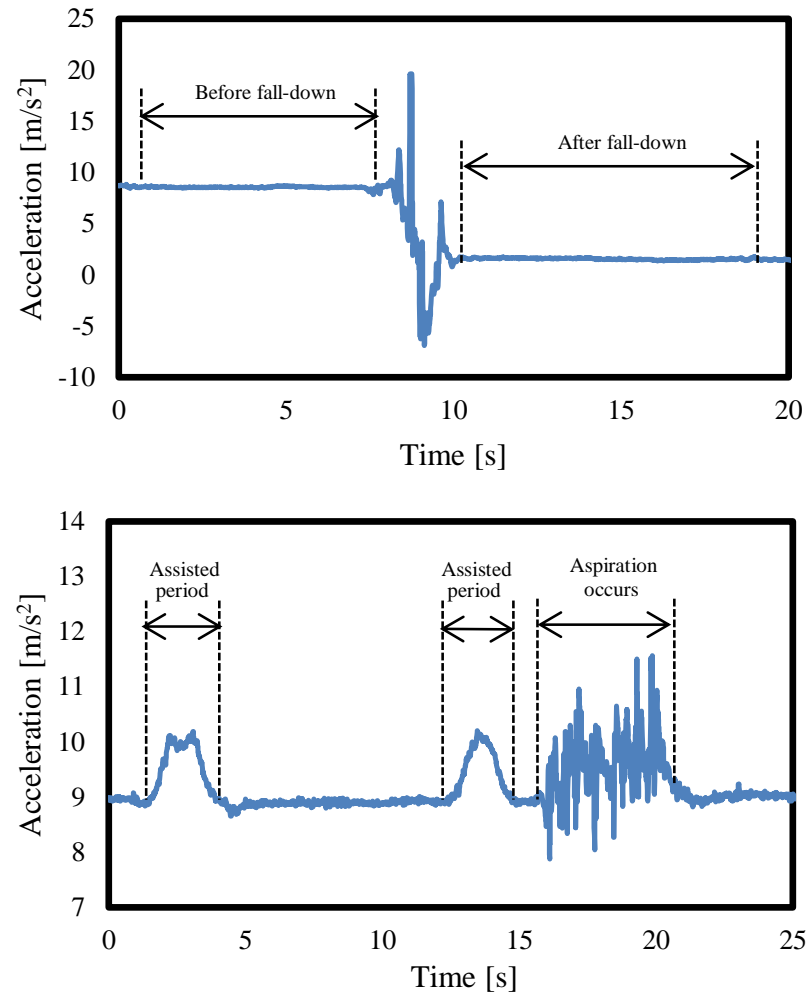

Fig. 7. Acceleration of y-axis as an aspiration occurs. 
As shown in Fig. 5, the acceleration changes as aspiration occurs. Therefore, by using the threshold of the value of the difference $|D|$ and for the duration time $t_{D}$, an aspiration can be detected. From the experimental rule, the threshold is set $|D| \geqq 0.4 \mathrm{~m} / \mathrm{s}^{2}, t_{D} \geqq 3 \mathrm{~s}$.

\subsection{Fall-down Detection}

Figure 6 shows the y-axis acceleration measurement when a person falling down from a chair to the right direction. As shown in Fig. 6, when falling down from a chair, as the device turns about 90 degree, the acceleration of gravity moves from $\mathrm{y}$-axis to either $\mathrm{x}$-axis or $\mathrm{z}$-axis, thus the measured acceleration for $y$-axis nears $0 \mathrm{~m} / \mathrm{s}^{2}$. This goes similarly when a person falls down towards any other direction besides right. Thus, by using $a_{y}$ and its duration time $t_{y}$ as the threshold, a fall-down accident can be detected. Considered the degree of the tilted chest and the degree tilted when falling to the floor is \pm 30 degree; the threshold of $\left|a_{y}\right| \leqq 4.9 \mathrm{~m} / \mathrm{s}^{2}, \quad t_{y} \geqq 3 \mathrm{~s}$ is set.

In addition, because both of the aspiration and a fall-down accident produce shock, there is a probability that the fall-down condition takes up the aspiration detection. However, as shown in Fig. 7, because of the y-axis of the acceleration of the aspiration is always $a_{y}>4.9 \mathrm{~m} / \mathrm{s}^{2}$, by identifying the $y$-axis acceleration, an aspiration and a fall-down accident can be distinguished.

\section{Evaluation Experiment}

\subsection{Purposed of Experiment}

This experiment is conducted to measure the recognition rate of the proposed system of detecting an aspiration and a fall-down accident.

Table 1. Results of experiment.

\begin{tabular}{|c|r|r|}
\hline \multirow{2}{*}{ Subjects } & \multicolumn{2}{|c|}{$\begin{array}{c}\text { Recognition Times } \\
\text { (success/test) }\end{array}$} \\
\cline { 2 - 3 } & Aspiration & \multicolumn{1}{c|}{ Fall-down } \\
\hline A & $10 / 10$ & $10 / 10$ \\
\hline B & $8 / 10$ & $10 / 10$ \\
\hline C & $10 / 10$ & $10 / 10$ \\
\hline D & $9 / 10$ & $10 / 10$ \\
\hline E & $10 / 10$ & $10 / 10$ \\
\hline
\end{tabular}

\subsection{Experimental Environment and Method}

The conducted experiment used an acceleration sensor built in Android smartphone FTJ152C (produced by FREETEL). The experiment is performed on 5 subjects. The smartphone was place upright on the subjects' chest and place sufficiently tight with a white band. After the smartphone sensor was turn on, the subjects intentionally simulated a fall-down accident 10 times continued by a period of spontaneous cough to resemble an aspiration incident. The successful detection of a fall-down accident and an aspiration was counted.

\subsection{Experimental Result and Study}

Table 1 shows the experimental result of the conducted experiment evaluated on 5 subjects. We confirmed that a fall-down accident and aspiration can be detected with high accuracy as shown in the experimental result. The maximum error when detecting an aspiration was two times in a tenth. We considered that this error was due to the short period of the cough of the subject. However, this error can hardly occur on real-life situation of an aspiration occurrence.

Therefore, the effectiveness of the purposed fall-down and aspiration detection by using the proposed system was confirmed.

\section{Conclusions}

In this paper, in conjunction with a meal assistance robot, a danger states detection system using an Android smartphone is proposed. The danger states are aspiration and a fall-down accident. The method of the detection of the danger state is by calculating the changes of the acceleration of the Android smartphone. This method system is installed in a smartphone and an experiment to measure its recognition rate is performed. As a result, the proposed system successful rate of detecting a fall-down accident and an aspiration was above 90\%. The experimental result shows that the proposed method is effective for both aspiration and fall-down detection.

Even so, intentional coughs were substituted in the experiment to test an aspiration. Therefore, as a future task, a field tests on an actual dietary behavior with a meal assistance robot will be performed. Thus, more detailed on the method's effectiveness and the smartphone's battery performance can be examined. 


\section{Acknowledgment}

This work was supported by JSPS KAKENHI Grant Numbers JP15K21197, JP15K12475.

\section{References}

(1) Statistic Bureau, Government of Japan : "Population Estimates", 2016

(2) Cabinet Office, Government of Japan : “Annual Report on the Aging Society 2015", 2015

(3) Miristry of Health, Labour and Welfare : "Transiton of number of registered care workers", 2014

(4) H. Tomimoto, M. Kirihara, S. Mu, S. Nakashima, K. Tanaka, K. Haruyama : "Meal Assistance Robot with Blink Operation Interface", Proceedings of the 4th IIAE International Conference on Industrial Application Engineering 2016, Vol. 27, No. 4, pp. 297-302, 2016

(5) H. Tomimoto, K. Tanaka, M. Kirihara, S. Nakashima and K. Haruyama : "Eye-Operated Meal Assistance Robot for Upper Limb Disorder", The society of life support engineering, Vol. 27, No. 4, pp. 132-137, 2015

(6) Guodong Feng, Jiechao Mai, Zhen Ban, Xuemei Guo, Guoli Wang : "Floor Pressure Imaging for Fall Detection with Fiber-Optic Sensors", IEEE Pervasive Computing, Vol. 15, No. 2, pp. 40-47, 2016

(7) Qu Chang, Sun Jie,Wang Junze, Zhu Xiaolong : "Automatic fall detection for the elderly using kinect sensor", Chinese Journal of Sensors and Actuators, Vol. 29, No. 3, pp. 378-383, 2016

(8) Wei-Han Chen, Hsi-Pin Ma: "A fall detection system based on infrared array sensors with tracking capability for the elderly at home", 2015 17th International Conference on E-Health Networking, Application and Services, No. 7454538, pp. 428-434, 2016

(9) Cuong Pham : "Mobicough: Real-time cough detection and monitoring using low-cost mobile devices", Intelligent Information and Database Systems, Vol. 9621, pp. 300-309, 2016

(10) Marcel Mlynczak, Katarzyna Pariaszewska, Gerard Cybulski: "Automatic cough episode detection using a vibroacoustic sensor", 2015 37th Annual International Conference of the IEEE Engineering in Medicine and Biology Society, Vol. 2015-12, No. 7318975, pp. 2808-2811, 2015 